\section{Comparative analysis of ChAd0x1 nCoV-19 and Ad26.COV2.S SARS-CoV-2 vector vaccines}

\author{
Stephan Michalik, ${ }^{1 *}$ Florian Siegerist,$^{2 *}$ Raghavendra Palankar, ${ }^{3 *}$ Kati \\ Franzke, ${ }^{4 *}$ Maximilian Schindler, ${ }^{2}$ Alexander Reder, ${ }^{1}$ Ulrike Seifert, ${ }^{5}$ Clemens \\ Cammann, ${ }^{5}$ Jan Wesche,${ }^{3}$ Leif Steil, ${ }^{1}$ Christian Hentschker, ${ }^{1}$ Manuela Gesell- \\ Salazar, ${ }^{1}$ Emil Reisinger, ${ }^{6}$ Martin Beer, ${ }^{7 \#}$ Nicole Endlich, ${ }^{2 *}$ Andreas \\ Greinacher $^{3 \#}$ and Uwe Völker ${ }^{1 \#}$
}

${ }^{1}$ Interfaculty Institute of Genetics and Functional Genomics, Department of Functional Ge-nomics, University Medicine Greifswald, Greifswald; ${ }^{2}$ Institute for Anatomy and Cell Biology, University Medicine Greifswald, Greifswald; ${ }^{3}$ Institute of Transfusion Medicine, University Medicine Greifswald, Greifswald; ${ }^{4}$ Institute of Infectiology, Friedrich-LoefflerInstitut, Greifswald-Insel Riems; ${ }^{5}$ Friedrich Loeffler-Institute of Medical MicrobiologyVirology, University Medicine Greifswald, Greifswald; ${ }^{6}$ Division of Tropical Medicine and Infectious Diseases, Center of Internal Medicine II, Rostock University Medical Center, Rostock, and ${ }^{7}$ Institute of Diagnostic Virology, Friedrich-Loeffler-Institut, Greifswald-Insel Riems, Germany

$\star S M, F S, R P$ and KF contributed equally as co-first authors

${ }^{\#} M B, N E, A G$ and UV contributed equally as co-senior authors

\section{ABSTRACT}

$\mathrm{V}$ ector-based SARS-CoV-2 vaccines have been associated with vaccine-induced thrombosis with thrombocytopenia syndrome (VITT/TTS), but the causative factors are still unresolved. We comprehensively analyzed the ChAdOx1 nCoV-19 (AstraZeneca) and Ad26.COV2.S (Johnson \& Johnson) vaccines. ChAdOx1 nCoV-19 contains significant amounts of host cell protein impurities, including functionally active proteasomes, and adenoviral proteins. A much smaller amount of impurities was found in Ad26.COV2.S. Platelet factor 4 formed complexes with ChAdOx1 nCoV-19 constituents, but not with purified virions from ChAdOx1 nCoV-19 or with Ad26.COV2.S. Vascular hyperpermeability was induced by ChAdOx nCoV-19 but not by Ad26.COV2.S. These differences in impurities together with EDTAinduced capillary leakage might contribute to the higher incidence rate of VITT associated with ChAdOx1 nCoV-19 compared to Ad26.COV2.S.

\section{Introduction}

Vaccination is key for the control of the severe acute respiratory syndrome coronavirus-2 (SARS-CoV-2) pandemic. Adenoviral vector-, mRNA encapsulated in lipid nanoparticles-, and antigen-based vaccines are currently in use, all encoding the spike protein. ${ }^{1,2}$ Since February 2021 the rare but severe adverse reaction of vaccine-induced immune thrombotic thrombocytopenia (VITT; synonym thrombosis with thrombocytopenia syndrome [TTS]) has been observed in individuals vaccinated against SARS-CoV-2. VITT/TTS occurs 5-20 days (occasionally later) after vaccination with the ChAdOx1 nCoV-19 vaccine (produced by AstraZeneca) and the Ad26.COV2.S vector vaccine (produced by Janssen/Johnson \& Johnson). The incidence rate of VITT/TTS seems to be higher for ChAdOx1 nCoV-19. The reported rate of VITT/TTS in the USA is 0.355 cases per 100,000 people vaccinated with Ad26.COV2.S, ${ }^{3}$ compared to 1 per 50,000-100,000 people vaccinated with ChAdOx1 $\mathrm{nCoV}-19$ in the UK. ${ }^{4}$ In Germany both vaccines were used and within this medical system, 0.56 suspected cases per 100,000 vaccine doses for Ad26.COV2.S (3,186,297 vaccine doses administered) and 1.49 suspected cases per 100,000 vaccine doses for ChAdOx1 nCoV-19 (12,692,700 vaccine doses administered) were reported. ${ }^{5}$ VITT/TTS involves high-affinity, platelet-activating anti-platelet factor 4 (PF4) antibodies, ${ }^{6-8}$ but the mechanisms triggering these anti-PF4 antibodies are still unre-
Ferrata Storti Foundation

Haematologica 2022

Volume 107(4):947-957

\section{Correspondence:}

ANDREAS GREINACHER,

andreas.greinacher@med.uni-greifswald.de

UWE VÖLKER

voelker@uni-greifswald.de

Received: October 5, 2021.

Accepted: January 5, 2022.

Pre-published: January 20, 2022.

https://doi.org/10.3324/haematol.2021.280154

(C)2022 Ferrata Storti Foundation

Material published in Haematologica is covered by copyright. All rights are reserved to the Ferrata Storti Foundation. Use of published material is allowed under the following terms and conditions:

https://creativecommons.org/licenses/by-nc/4.0/legalcode. Copies of published material are allowed for personal or internal use. Sharing published material for non-commercial purposes is subject to the following conditions:

https://creativecommons.org/licenses/by-nc/4.0/legalcode, sect. 3. Reproducing and sharing published material for commercial purposes is not allowed without permission in writing from the publisher. 
solved. VITT/TTS shows striking similarities with another PF4-mediated adverse drug effect, heparin-induced thrombocytopenia (HIT) and autoimmune HIT. In HIT, polyanions form complexes with PF4, inducing a conformational change, which triggers anti-PF4 antibodies. This immune response is pronounced in patients with tissue trauma and inflammation. We have shown that one or more constituents of the ChAdOx1 $\mathrm{nCoV}-19$ vaccine interact with PF4, forming complexes which contain PF4 and the adenovirus hexon protein. This might trigger conformational changes in the positively charged chemokine PF4 leading to the formation of a neo-antigen and then subsequent activation of B cells in a pro-inflammatory environment. ${ }^{9}$ These activated B cells then produce high avidity anti-PF4 antibodies that bind PF4 and trigger an activation cascade of platelets and granulocytes, leading to NETosis and massive thrombin production. However, it is not known which vaccine components, beside the hexon protein, interact with PF4 and which additional factors influence this interaction.

Both vaccines (ChAdOx1 nCoV-19 and Ad26.COV2.S) are produced in human cell lines, T-REx-293 cells (human embryonic kidney cells, a HEK293 derivate) for ChAdOx1 nCoV-19 and PER.C6 TetR cells (human embryonic retinal cells) for Ad26.COV2.S. We and others have previously shown that the ChAdOx $1 \mathrm{nCoV}-19$ vaccine contains a large number of host cell proteins (HCP)., ${ }^{9,10}$ Here we report the results of a comprehensive, comparative analysis of the ChAdOx1 nCoV-19 and Ad26.COV2.S vaccines, using proteomics, transmission electron microscopy, dynamic lightscattering, single-molecule light microscopy, and an in vivo capillary leakage assay.

Our data reveal substantial differences in composition and functional properties between the two vaccines, which may contribute to the different incidences of VITT/TTS.

\section{Methods}

Comprehensive details of the Methods are described in the Online Supplementary Material.

All experiments were performed in accordance with local and national ethics standards and German animal protection legislation, overseen by the "Landesamt für Landwirtschaft, Lebensmittelsicherheit und Fischerei, Rostock" of the federal state of Mecklenburg - Western Pomerania.

\section{Sample preparation and liquid chromatography tandem mass spectrometry.}

Vaccines were precipitated using salt-acetone precipitation. ${ }^{11}$ Adenovirus particles were purified using subsequent sucrosecushion and sucrose-gradient ultracentrifugation. Protein was digested with trypsin as described by Blankenburg et al. ${ }^{12}$ Liquid chromatography tandem mass spectrometry (LC-MS/MS) experiments were performed on an Orbitrap Exploris ${ }^{\mathrm{TM}} 480$ mass spectrometer (Thermo Scientific, Bremen, Germany) coupled to an Ultimate $^{\mathrm{TM}} 3000$ RSLCnano HPLC (Dionex/ Thermo Scientific, Waltham, MA, USA). The mass spectrometry proteomics data have been deposited with the ProteomeXchange Consortium via the PRIDE18 partner repository ${ }^{13}$ with the dataset identifier PXD027344.

\section{Sodium dodecylsulfate gel electrophoresis and western blot analysis}

For protein separation, one-fiftieth of one vaccine dose, as well as dilutions of HEK293 total protein lysate, were loaded onto a precast NuPAGE ${ }^{\mathrm{TM}} 4$ to $12 \%$ gel. Electrophoresis was performed at $150 \mathrm{~V}$. The western blots were prepared using the Trans-Blot Turbo Transfer System from BioRad and the transfer of proteins to the polyvinylidene fluoride membrane was verified and documented using LICOR's Revert Total Protein Stain protocol (Doc \# 988-19494). The specific proteins were detected with primary and secondary antibodies described in the Online Supplementary Methods.

\section{Proteasome activity assays}

Chymotrypsin-like activity was assessed in vaccine or HEK293T cell lysate using $0.2 \mathrm{mM}$ fluorescently tagged Suc-LLVYAMC (Bachem, Bubendorf, Switzerland) quantified with a fluorometer using a 380/460 $\mathrm{nm}$ filterset. To confirm proteasomal activity $100 \mathrm{nM}$ bortezomib ${ }^{14}$ or $200 \mathrm{nM}$ carfilzomib (Selleckchem, Houston, TX, USA) ${ }^{15}$, was added.

\section{Dynamic light scattering and zeta potential}

All dynamic light scattering measurements were performed in a fixed scattering angle Zetasizer Nano-S system (Malvern Instruments Ltd., Malvern, UK). The hydrodynamic diameter (nm) was measured at $25^{\circ} \mathrm{C}$, and light scattering was detected at $173^{\circ}$. Surface $\zeta$ potential was performed in folded capillary $\zeta$ cells (DTS1070, Malvern Instruments Ltd., Malvern, UK). Data were analyzed using Zetasizer software, version 7.13 (Malvern Instruments Ltd., Malvern, UK).

\section{Immunoelectron and transmission electron microscopy}

Vaccines or the purified adenovirus particles were incubated with biotinylated PF4 and transferred to formvar-coated transmission electron microscopy grids. After washing, samples were labeled with an anti-adenovirus monoclonal antibody detected by a gold conjugate. The same samples were labeled with a streptavidin-gold conjugate to detect PF4-biotin. Grids were stained with $1 \%$ phosphotungstic acid and analyzed with a Tecnai-Spirit transmission electron microscope (FEI, Eindhoven, Germany).

\section{Super resolution single-molecule light microscopy}

Diluted vaccine or purified virions were incubated with human PF4 and immobilized on cleaned coverslips. After fixation and blocking, PF4 and adenoviral hexon were visualized using secondary (PF4) and primary (hexon) immunofluorescence detected by Alexa Fluor 488 and Cy5. Coverslips were mounted in Everspark dSTORM buffer (Idylle Labs, France) ${ }^{16}$ and blinking sequences imaged on a Zeiss Elyra PS.1 super resolution system. Single-molecule localization microscopy data were processed in FIJI using NanoJ core $^{17}$ and Thunderstorm ${ }^{18}$ and analyzed using custom FIJI ${ }^{19}$ scripts.

\section{Zebrafish vascular permeability assay}

A novel zebrafish-based in vivo assay was developed to determine local changes of vascular permeability following intramuscular injections: Transgenic zebrafish at 5 days post-fertilization expressing a $78 \mathrm{kDa}$ GFP-tagged plasma protein ${ }^{20,21}$ were injected intramuscularly with $1 \mathrm{~nL}$ of native vaccine, purified virions, 100 $\mu \mathrm{M}$ EDTA or $0.9 \% \mathrm{NaCl}$. Fluorescence intensity ratios (intravascular $v s$. intramuscular) were measured in the direct vicinity of the injection site at $\mathrm{t}=0$ and $\mathrm{t}=10 \mathrm{~min}$.

\section{Results}

Comparative profiling of ChAd0x1 nCoV-19 and Ad26.COV2.S vaccines

Comparative profiling of $\mathrm{ChAdOx} 1 \mathrm{nCoV}-19$ and 
Ad26.COV2.S (three different lots each) consistently revealed significant differences: (i) the total protein concentration of the ChAdOx1 nCoV-19 vaccine was approximately 3.4-times higher than that of the Ad26.COV2.S vaccine (mean: $102 \mathrm{ng} / \mu \mathrm{L}$ vs. $29.8 \mathrm{ng} / \mu \mathrm{L}$ ) (Figure 1A); (ii) silvernitrate staining of vaccines separated by sodium dodecylsulphate polyacrylamide gel electrophoresis (SDS-PAGE) displayed a markedly more complex protein pattern than expected for pure virions for $\mathrm{ChAdOx} 1 \mathrm{nCoV}-19$ compared to Ad26.COV2.S (Figure 1B). (iii) mass spectrometric analysis (Online Supplementary Table S1) identified a much higher proportion ( $44.5 \%$ to $59.2 \%$ vs. only $0.26 \%$ to $0.96 \%$ ) (Figure 1D, Online Supplementary Figures $S 1$ and S2) and

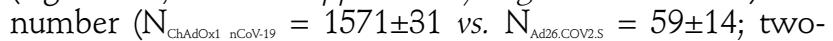
sided $t$-test $\left.P=8.709 \times 10^{-6}\right)$ (Online Supplementary Figure S2) of host cell-derived human proteins. A dilution series of a laboratory HEK293 cell line lysate, which was used instead of the cell line used for vaccine production, confirmed the quantities of host-cell proteins $(54 \%$ for ChAdOx $1 \mathrm{nCoV}$ 19 and 1.5\% for Ad26.COV2.S) (Online Supplementary Figure S3). None of the top ten most abundant human proteins in ChAdOx1 nCoV-19 was detected in Ad26.COV2.S (Online Supplementary Table S2). Adenoviral proteins accounted for 40.8-55.5\% (ChAdOx1 nCoV-19) and 99.04-99.74\% (Ad26.COV2.S) of total ion intensity, while the SARS-CoV2 spike protein was detected only in the ChAdOx1 nCoV19 vaccine (3 different lots) (Online Supplementary Figure S1). Western blot analysis confirmed the significant abundance of 11 selected host-cell proteins in the ChAdOx1 nCoV-19 vaccine, which even exceeded the abundances detected in
A

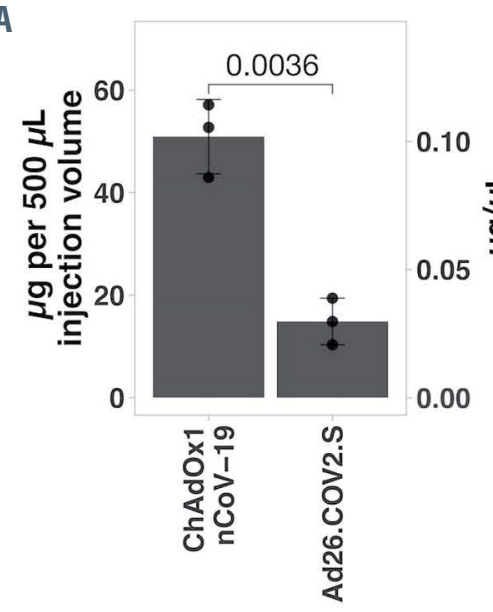

B

HEK293 $(\mu \mathrm{g})$

ChAdOx1 Ad26. nCoV-19 COV2.S

\section{M}

เก 0 เก เึ
C
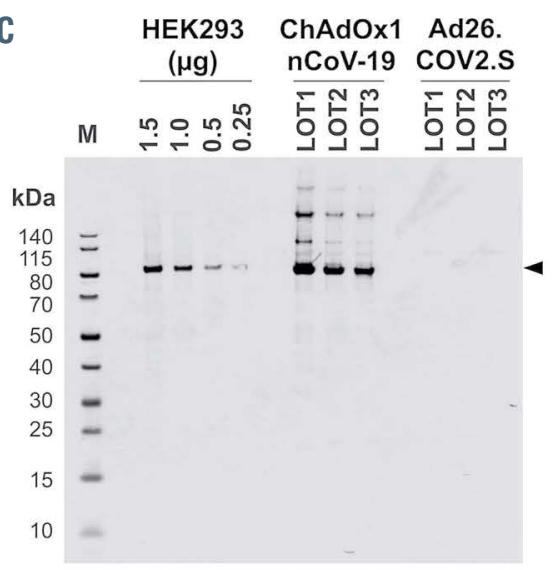

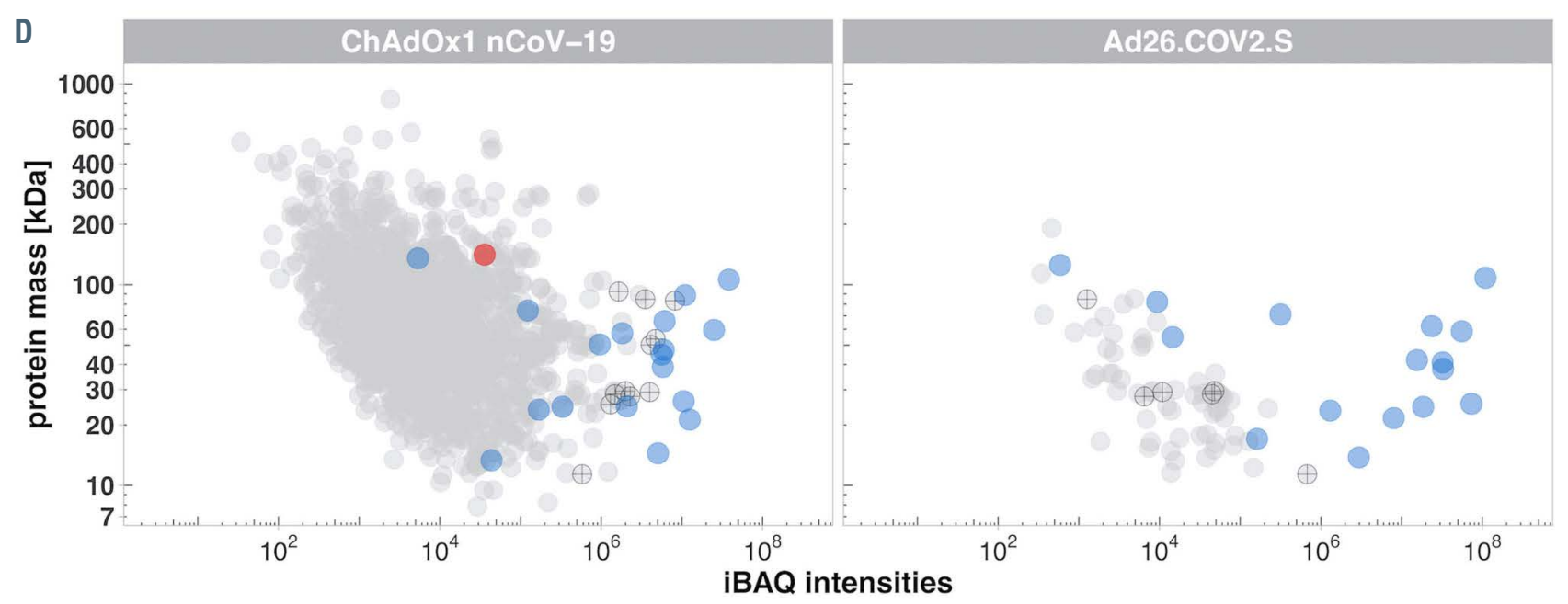

human protein SARS-CoV-2 spike protein vector protein

Figure 1. Analysis of the protein composition of ChAdOx1 nCoV-19 and Ad26.cov2.S vaccines. (A) Determination of the protein concentration of the two vaccines ( 3 different lots each). Protein concentration was determined with a quantitative bicinchoninic acid (BCA) assay. Protein concentration per $500 \mu \mathrm{L}$ (vaccination dose) and $1 \mu \mathrm{L}$ vaccine (secondary axis) of three lots of ChAdOx1 nCoV-19 or Ad26.COV2.S vaccine, respectively, are shown. Statistical testing was performed using a twosided $t$-test. (B) Protein patterns of silver nitrate-stained sodium dodecylsulfate polyacrylamide gel electrophoresis of ChAdOx1 nCoV-19 or Ad26.COV2.S (3 lots each) vaccines along with a dilution series of a laboratory HEK293 cell line extract for comparison. The HEK293 cell extract was loaded onto the gel at 1.5, 1.0, 0.5, or $0.25 \mu \mathrm{g}$ per lane, and $10 \mu \mathrm{L}\left(1 / 50^{\text {th }}\right.$ of a vaccine dose) were used for each vaccine. (C) Western blot analysis of HSP90- $\alpha$ protein, using the same gel loading scheme as for the silver nitrate-stained gel. (D) intensity-based absolute quantification (iBAQ) protein intensities and theoretical molecular mass of identified proteins. Protein intensities of ChAdOx1 nCoV-19 or Ad26.COV2.S (exemplarily shown for lot 3) were calculated using the iBAQ algorithm (minimum of 3 unique peptides per protein) and plotted against the theoretical molecular mass. Proteins are color-coded according to their respective class. Blue dots indicate vector proteins; gray dots represent human proteins; the red dot indicates the SARS-CoV-2 spike protein. Points highlighted with a cross indicate proteins additionally analyzed by western blotting in Online Supplementary Figures S4-S7. 
the HEK293 cell line. None of these proteins was detected in the Ad26.COV2.S vaccine (Figure 1C, Online Supplementary Figure S4).

In summary, per vaccine dose $(500 \mu \mathrm{L})$ of ChAdOx1 $\mathrm{nCoV}-19$ we detected 19.1-33.8 $\mu \mathrm{g}$ host-cell proteins and 23.3-26.3 $\mu \mathrm{g}$ chimpanzee adenovirus proteins and for the Ad26.COV2.S vaccine 0.04-0.19 $\mu \mathrm{g}$ host-cell proteins and 10.2-19.2 $\mu \mathrm{g}$ adenoviral proteins. Since the approximately $5 \times 10^{10}$ virions per vaccine dose weigh about $12.5 \mu \mathrm{g}$, both vaccines contain unassembled virus proteins, mostly hexon proteins. However, the amount of approximately 10-14 $\mu \mathrm{g}$ unassembled virus proteins in ChAdOx1 nCoV-19 was again much larger compared to the $0-6.5 \mu \mathrm{g}$ in Ad26.COV2.S.

\section{Proteasome activity in the different vaccines}

Proteasome subunits were identified by mass spectrometry and verified by western blot analysis (Figure 2A, Online Supplementary Figure S5). Chymotrypsin-like activity associated with the proteasomal $\beta-5$ subunit showed lot-dependent high levels in ChAdOx1 nCoV-19, while in Ad26.COV2.S only minimal proteasome activity was found in one of three lots (Figure 2B, C). Substrate turnover

A

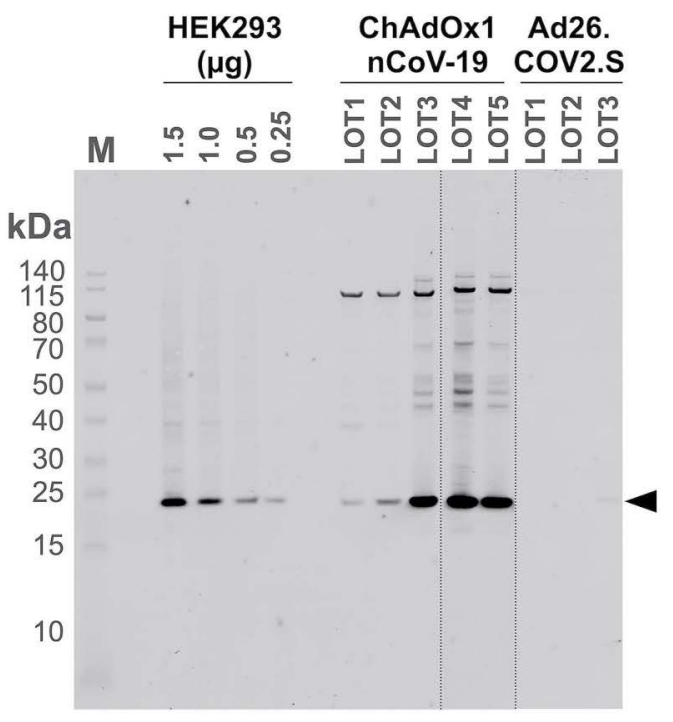

varied in the different lots according to the proteasome subunit expression level (Figure 2A, C). Inhibition of the proteasome activity by $100 \mathrm{nM}$ bortezomib ${ }^{14}$ or $200 \mathrm{nM}$ carfilzomib $^{15}$ confirmed assay specificity (Online Supplementary Figure S6).

\section{Platelet factor 4-vaccine cluster formation}

PF4 is the key protein involved in the immune response causing VITT. We assessed the interaction of PF4 with the native vaccines and the purified adenoviral particles of ChAdOx1 nCoV-19, which were obtained by sucrose cushion and gradient ultracentrifugation. The purity of isolated ChAdOx1 nCoV-19 virions was confirmed by transmission electron microscopy and silver-staining of one-dimensional SDS-PAGE (Online Supplementary Figures S7 and S8).

Dynamic light scattering confirmed PF4-induced clustering of the non-purified ChAdOx1 $\mathrm{nCoV}-19$ vaccine (Figure $3 \mathrm{~A}$, left panel). The hydrodynamic diameter increased from $88 \pm 2.4 \mathrm{~nm}$ up to $151 \pm 12 \mathrm{~nm}$ and $320 \pm 45 \mathrm{~nm}$ with 10 $\mu \mathrm{g} / \mathrm{mL}$ and $50 \mu \mathrm{g} / \mathrm{mL}$ PF4, respectively (Figure 3A; the PF4 dose-dependent size change is shown in Online Supplementary Figure S9). This complex formation was reversible upon the addition of unfractionated heparin and

B

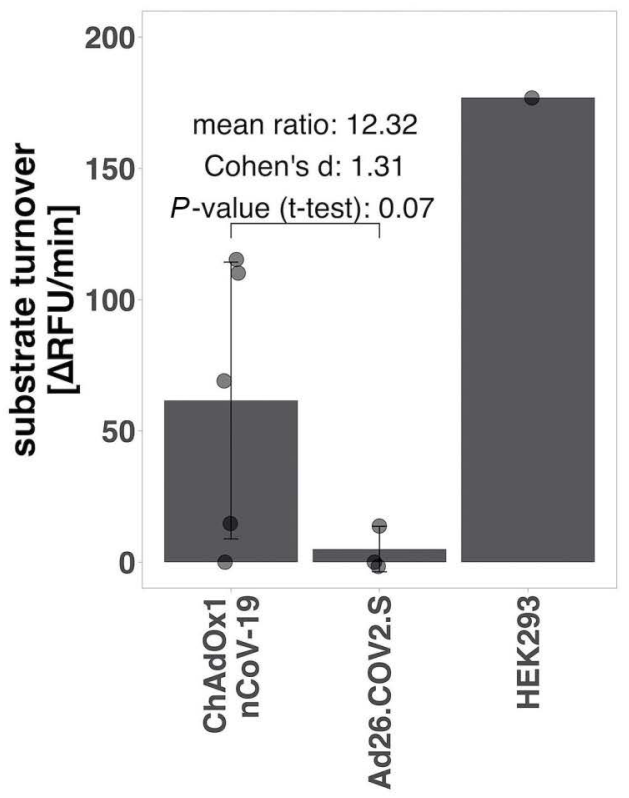

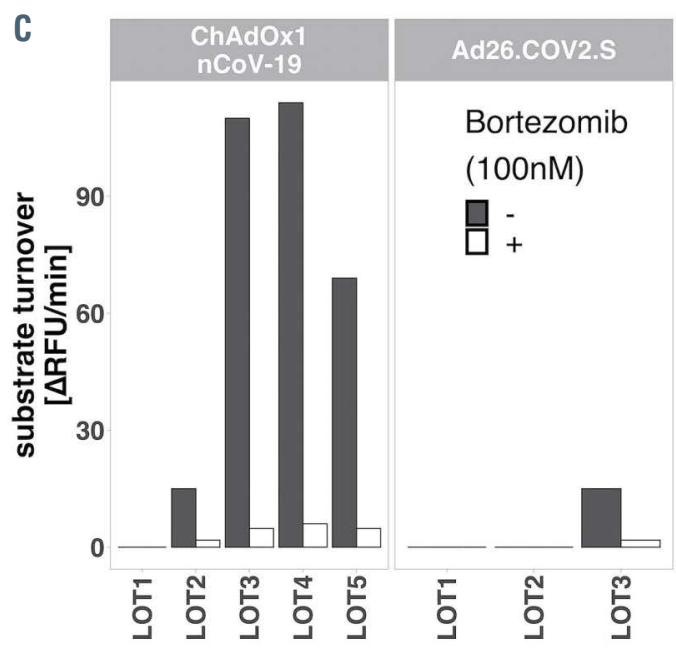

Figure 2: Analysis of proteasome proteins and activity in the vaccines. (A) Western blot analysis of proteasome $20 \mathrm{~S}$ subunit beta 5 (the original western blot image is provided in Online Supplementary Figure S4). (B) Fifty microliters $\left(1 / 10^{\text {th }}\right.$ of the vaccination dose) of different ChAdOx1 nCoV-19 $(n=5)$ and Ad26.COV2.S $(n=3)$ lots were analyzed for the chymotrypsin-like activity of the proteasome and compared with the activity of $0.25 \mu \mathrm{g}$ of HEK293 cell lysate. The mean \pm standard deviation of two technical replicates are shown. (C) Proteasomal activity was confirmed by inhibition with $100 \mathrm{nM}$ bortezomib. The mean of two technical replicates is shown. 
can be attributed to weakened electropositive surface potential of PF4 in the presence of highly negatively charged unfractionated heparin thus decreasing its ability to interact electrostatically with vaccine components. In contrast, the addition of PF4 $(50 \mu \mathrm{g} / \mathrm{mL})$ only marginally increased the particle size when incubated with purified virions from ChAdOx1 nCoV-19 (from 79.6 $\pm 10.3 \mathrm{~nm}$ to $86.7 \pm 6.22 \mathrm{~nm} ; P=0.2404$ ) (Figure $3 \mathrm{~A}$, middle panel) or the Ad26.COV2.S vaccine (from $85.7 \pm 2.2 \mathrm{~nm}$ to $91.3 \pm 2.83 \mathrm{~nm}$; $P=0.0620$ ) (see particle size-frequency distribution plots in Online Supplementary Figure S10).

Complex formation of PF4 with the ChAdOx1 nCoV-19 vaccine was charge-dependent, as the negative charge of ChAdOx1 nCoV-19 ( $\zeta$ potential $-27.5 \pm 4.7$ ) was neutralized by PF4. In comparison, both, purified ChAdOx1 nCoV-19 virions $(\zeta$ potential $-1.7 \pm 4.7)$ and the untreated Ad26.COV2.S vaccine ( $\zeta$ potential $-4.5 \pm 5.7$ ) showed only a minimal negative charge (Figure $3 \mathrm{~B}$ ).

Consistent with dynamic light scattering findings and as described before, ${ }^{6}$ PF4 induced the formation of electrondense aggregates with ChAdOx1 nCoV-19 (Figure 4A), which contained unassembled hexon proteins (Online Supplementary Figure S11). In contrast, no comparable aggregates were detected after incubation of PF4 with purified virions from ChAdOx1 nCoV-19 (Figure 4B) or the Ad26.COV2.S vaccine (Figure 4C).

PF4 single-molecule density analysis using single-molecule localization microscopy (Online Supplementary Figures $S 12$ and S13) revealed that PF4 clusters formed on or in close vicinity to $\mathrm{ChAdOx} 1 \mathrm{nCoV}-19$ adenoviral hexon pro-

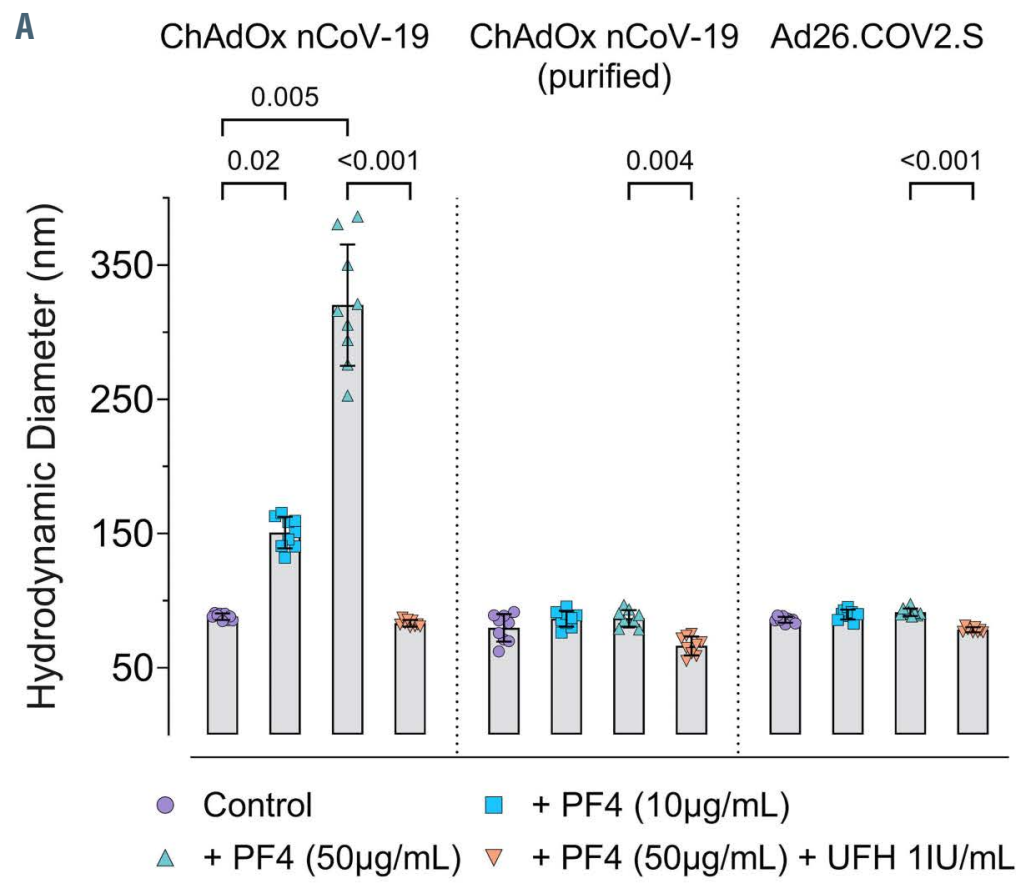

B

ChAdOx nCoV-19 ChAdOx nCoV-19 Ad26.COV2.S (purified)

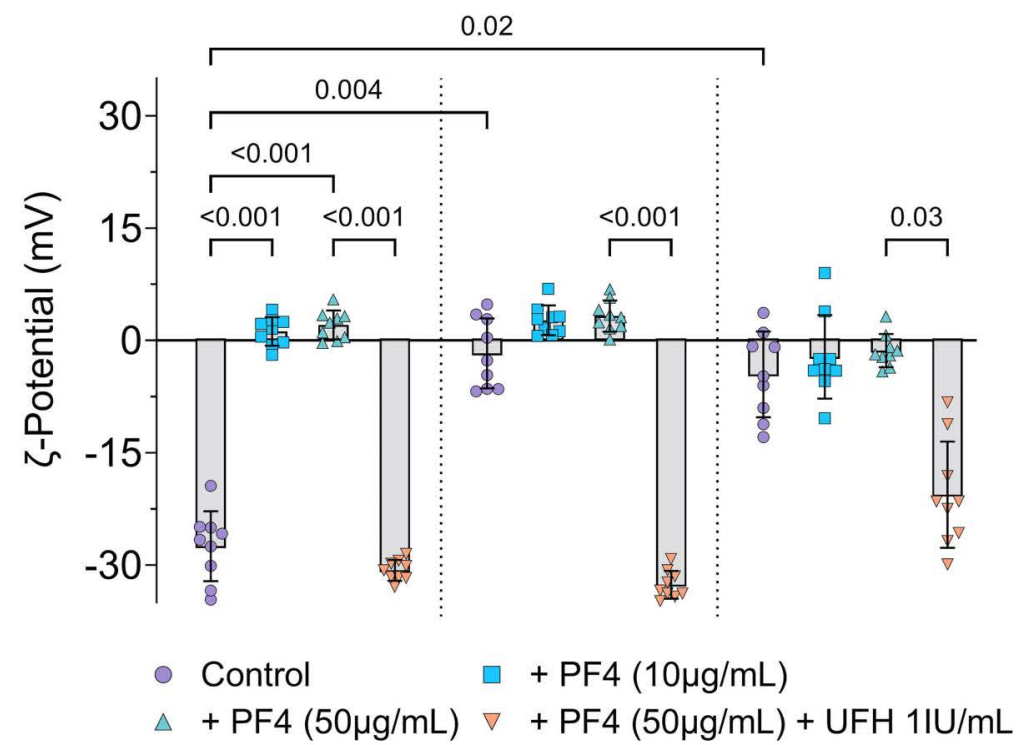

Figure 3. Dynamic light scattering analysis of vaccineinduced platelet factor 4 clustering. Analysis of ChAdOx1 nCoV-19 vaccine, purified ChAdOx1 nCoV-19 virions and Ad26.COV2.S vaccine by dynamic light scattering. (A) The hydrodynamic diameter (mean \pm standard deviation [SD], $\mathrm{n}=9$ ) of ChAdOx1 nCoV-19 or Ad26.COV2.S particles before and after addition of 10 $\mu \mathrm{g} / \mathrm{mL}$ or $50 \mu \mathrm{g} / \mathrm{mL}$ platelet factor 4 (PF4) was determined. A dose-dependent increase in the size of ChAdOx1 nCoV-19 aggregates in the presence of PF4 was detected. This effect was markedly reduced for both purified ChAdOx1 nCoV-19 virions and Ad26.COV2.S. Addition of unfractionated heparin (UFH; $1 \mathrm{lU} / \mathrm{mL}$ ) dissociated complexes between PF4 and vaccine components. (B) The $\zeta$-potential (mean \pm $\mathrm{SD}, \mathrm{n}=9$ ) of ChAdOx1 nCoV-19 was lower than the purified ChAdOx1 nCoV-19 virions or of Ad26.COV2.S; and largely neutralized by PF4. In the presence of UFH, a charge reversal to net negative charge was observed in both vaccines and purified virions from ChAdOx 1 nCoV-19 vaccine. Statistical analysis was performed by one-way analysis of variance on ranks/KruskalWallis followed by correction for multiple comparison by two-stage Benjamini, Krieger, \& Yekutieli controlling the false discovery rate procedure $(n=9)$ and $\alpha<0.05$ was considered significant. 
teins (PF4 single-molecule density ratio 7.09 \pm 1.38 , Figure 4D, G; arrowheads in Online Supplementary Figure S12), but not on Ad26.COV2.S $(1.13 \pm 0.14, P<0.0001)$ or purified ChAdOx1 nCoV-19 virions $(2.33 \pm 0.44, P=0.0115$, mean \pm standard error of mean; $P$-values refer to comparison with ChAdOx1 nCoV-19) (Figure 4D-G).

Dynamic light scattering experiments with ultracentrifugation-separated virions from the vaccines and the resulting supernatant confirmed the single-molecule density analysis of PF4 binding (Figure 5). Electron microscopy analysis revealed absence of intact virions in the supernatant fraction of both ChAdOx1 nCoV-19 and Ad26.COV2.S, while the pellet was enriched in virions (Figure 5A, B). Intriguingly, we observed amorphous electron-dense irregularly shaped particulate material in ChAdOx1 nCoV-19 supernatant that was absent in Ad26.COV2.S. No significant PF4-dependent complex formation was detected by dynamic light scattering with the pellet fractions of ChAdOx1 nCoV-19 and Ad26.COV2.S, but the supernatant fraction of ChAdOx1

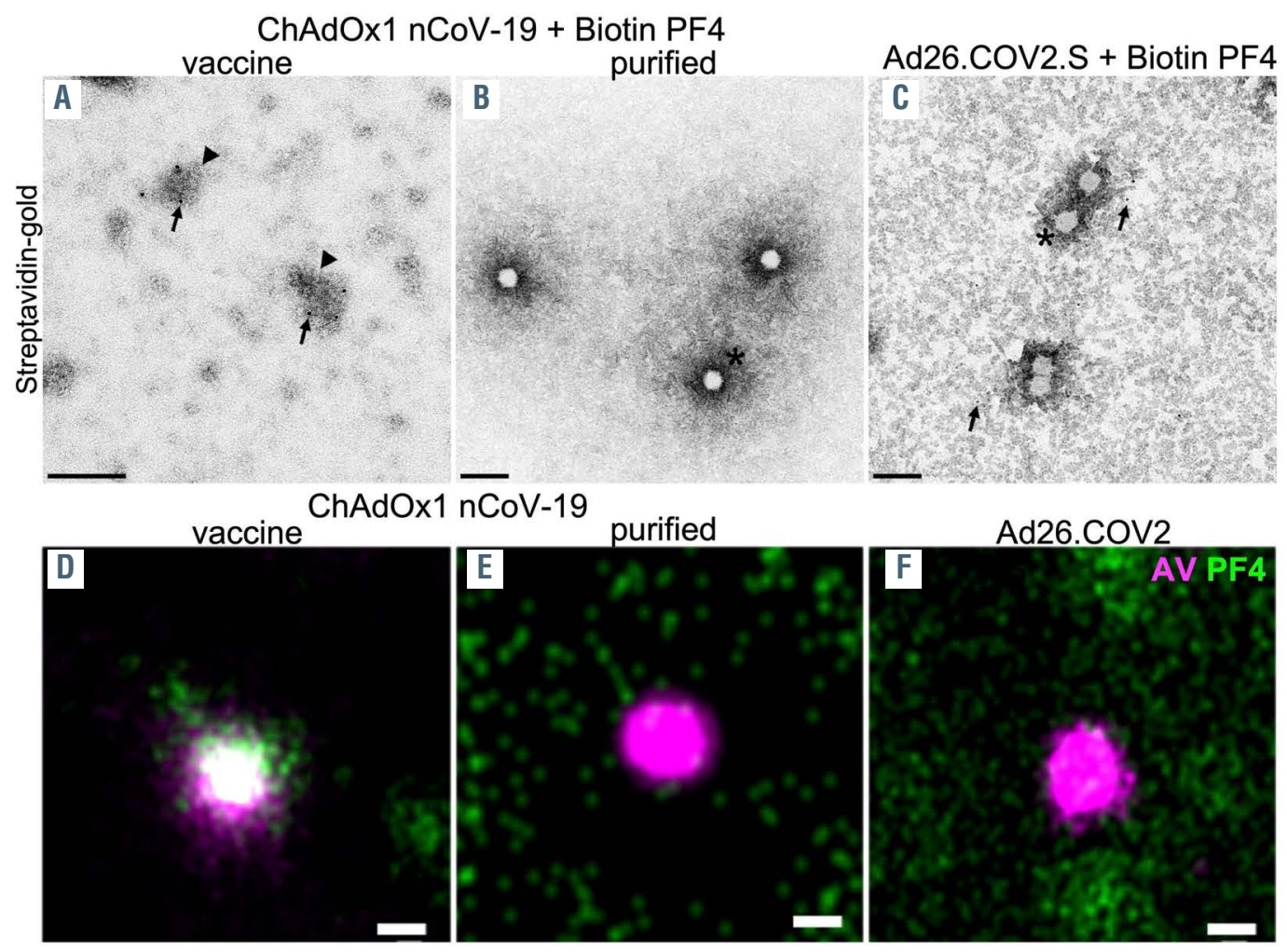

G

$<0.0001$
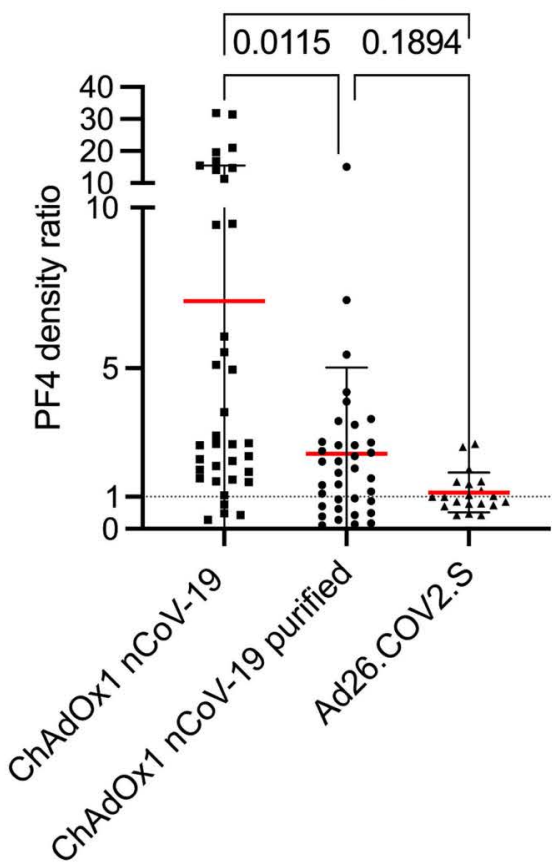

Figure 4. Ultrastructural analysis of vaccine-induced platelet factor 4 clustering (A-C) Representative micrographs of streptavidin-gold immunoelectron microscopy (arrow); biotinylated, human platelet factor 4 (PF4) was incubated with either ChAdOx1 nCoV-19 (A), purified virions from ChAdOx1 nCoV-19 (B) or Ad26.COV2.S (C). Virions are exemplarily labeled by asterisks, and immunogoldlabeled aggregates by arrowheads; scale bars represent $200 \mathrm{~nm}$. (D-F) Singlemolecule light microscopy show representative dual PF4 and hexon polypeptide reconstructions after incubation with either ChAdOx1 nCoV-19 (D), purified virions from ChAdOx1 nCoV-19 (E) or Ad26.COV2.S (F). As indicated by the green signal in close proximity to adenoviral particles in (D), PF4 was found in dense clusters after incubation with ChAdOx1 nCoV-19 whereas PF4 formed a more homogeneous layer on glass after incubation with purified ChAdOx1 nCoV-19 (E) or Ad26.Cov2.S (F). For quantification, single-molecule particle analysis was performed and particle density ratios (on viral particles/on glass) analyzed (Online Supplementary Figure S10). (G) Relative PF4 particle densities, showing a statistically significant affinity of PF4 to adenoviral particles predominantly on ChAdOx1 nCoV-19 but not on purified ChAdOx1 nCoV-19 virions or Ad26.COV2.S. Statistical analysis of $n=92$ particles was performed with the Kruskal-Wallis test with the Dunn correction for multiple comparisons. Respective $P$-values are indicated in the plot, red lines and whiskers indicate mean \pm standard deviation. The dashed line is at $y=1$ (equal affinity of PF4 for adenoviral hexon and glass). Full-field of view images and single-channel reconstructions are provided in Online Supplementary Figure S9. Scale bars represent $100 \mathrm{~nm}$. 
nCoV-19 showed clear PF4 complex formation potential (Figure 5B). However, it is important to note that under in vitro conditions, PF4 binds to both chimpanzee adenovirus Y25 (ChAdOx1) and human adenoviruses (HAdVD26 and HAdV-C5) through weak electrostatic interactions that were abrogated in the presence of fondaparinux, a heparin pentasaccharide ${ }^{22}$. Proteomic analysis showed that the host-cell protein content in the pellet fraction was reduced compared to the non-fractionated vaccine and a large proportion of the ChAdOx1 nCoV-19 host-cell proteins was located in the supernatant fraction. As expected, the viral proteins were enriched in the pellet fraction (Figure 5E, Online Supplementary Figures S14-S16).

\section{ChAd0x1 nCoV-19 induced vascular hyperpermeability}

To study the effect of the two vaccines and EDTA (100 $\mu \mathrm{M}$ present in the ChAdOx1 $\mathrm{nCoV} 19$ vaccine) on vascular permeability, we used in vivo microscopy of transgenic zebrafish larvae expressing an eGFP-tagged plasma protein (gc-eGFP, $78 \mathrm{kDa}^{20}$ ). Intramuscular injections (Online Supplementary Video S1) of $1 \mathrm{~nL}$ of $100 \mu \mathrm{M}$ EDTA or ChAdOx1 nCoV-19 locally increased vascular permeability, indicated by leakage of eGFP from the intravascular to the intramuscular compartment $(P=0.0001)$, but this was not observed after injection of purified ChAdOx1 nCoV19 virions, Ad26.COV2.S or physiological saline (Figure $6)$.

\section{Discussion}

Our comprehensive analyses revealed major differences between ChAdOx1 nCoV-19 and Ad26.COV2.S vaccines. Confirming our previous observation, a high proportion of host-cell proteins (54\%) was found in the ChAdOx1 nCoV-19 vaccine, but only a very low level was found in Ad26.COV2.S (1.5\%). This observation suggests very different purification approaches and purification efficiencies for the two vaccines, with a more thorough purification of adenoviruses in Ad26.COV2.S. Such differences might be caused by the use of detergent treatment of the infected production cell culture for ChAdOx1 nCoV-19, which will make subsequent purification strategies more complicated. ${ }^{23}$

The SARS-CoV-2 spike protein was detected only in the ChAdOx1 nCoV-19 vaccine. There may be several reasons for this, since different cell lines and procedures were used for production and purification of the two vaccines. Both suppliers use systems in which the TetR repressor suppresses the expression of the SARS-CoV-2 transgene during the production of the recombinant adenoviral vectors. A different degree of leakage of repression might occur in the cell lines thus allowing different residual expression of the spike protein. Another reason could be depletion of the spike protein during purification of Ad26.COV2.S. This can only be differentiated by inprocess sampling at different production steps of the vaccines.

The two vaccines display differences in their ability to interact with PF4. We confirm the previously observed complex formation of PF4 with ChAdOx1 nCoV-19. Consistent with a recent study using cryo-electron microscopy of ChAdOx1 nCoV-19, ${ }^{22}$ in our study addition of PF4 to purified ChAdOx1 nCoV-19 virions or to Ad26.COV2.S also resulted in a slight increase in particle size. This was reversed by addition of heparin, indicating charge-related binding of PF4 to the virions. This is again consistent with the data obtained by cryo-electron microscopy and in silico modeling of ChAdOx1 nCoV-19, which established a possible electrostatic interaction of positively charged PF4 and negatively charged adenovirus hexon polypeptide. ${ }^{22}$ However, the interaction $\mathrm{Kd}$ of about 300 nmol was rather weak. This is likely the reason that we could not demonstrate distinct complexes of PF4 with Ad26.COV2.S or purified ChAdOx1 nCoV-19 virion preparations. This further supports a role of impurities in ChAdOx1 nCoV-19 for the observed formation of large complexes in ChAdOx1 nCoV-19 after addition of PF4. We have shown that unassembled adenoviral hexon proteins are part of the PF4-complexes, but we still cannot exclude a contribution of additional constituents of the vaccine supernatant.

Antibody formation against PF4 is enhanced by antigen presentation in an inflammatory environment. Recently, we have shown (in collaboration with the laboratory of Prof. Thomas Renne, Universitätsmedizin Hamburg Eppendorf, Germany) that intradermal injection of ChAdOx1 nCoV-19 leads to EDTA-induced capillary leakage in the Mile skin edema assay, thus increasing the vaccine's intravascular distribution. ${ }^{9}$ Our zebrafish model allows intramuscular injection, which recapitulates the actual mode of vaccination. EDTA and $\mathrm{ChAdO} \times 1 \mathrm{nCoV}$ 19 but not Ad26.COV2.S rapidly induced local vascular hyperpermeability. Such an increase of local capillary leakage might facilitate direct contact of the immune system with vaccine components, as does accidental intravascular administration of the vaccine. ${ }^{24}$ Inflammation early after vaccination may also be enhanced when host-cell proteins are recognized by endogenous natural IgG. ${ }^{25}$ These natural antibodies bind proteins of degrading cells and can form immune complexes.

Furthermore, proteasome activity was detectable in both vaccines, again with a remarkable difference between Ad26.COV2.S (only low proteasome activity and protein abundance in one lot) compared to substantially higher proteasome activities in almost all lots of ChAdOx1 nCoV-19. This is of particular interest since Hauler et al. showed that adenoviral capsid proteins are intracellularly processed by proteasomal degradation which is mediated by the chaperone p97/VCP. ${ }^{26}$ In our proteomic analysis, we identified VCP as one of the top five most abundant proteins of the ChAdOx1 $\mathrm{nCoV}-19$ vaccine (Online Supplementary Tables $S 1$ and S2). Proteasomal degradation of adenoviral components such as the hexon polypeptide and/or host-cell proteins might lead to a reduction in vaccine efficiency. Whether proteasomal activity may also create potentially immunogenic or immunoreactive neo-antigens remains unresolved.

VITT/TTS occurs clinically after vaccination with ChAdOx1 nCoV-19 or Ad26.COV2.S and is mediated by platelet-activating anti-PF4 antibodies. Recently, we and others have shown that anti-spike protein antibodies and anti-PF4 antibodies react independently of each other, therefore making it unlikely that VITT is caused by crossreacting anti-spike protein antibodies. ${ }^{27,28}$ The current data indicate that adenovirus particles and free hexon proteins are the common features of both vaccines. We only observed formation of larger complexes of PF4 with ChAdOx1 nCoV-19. This indicates that an additional 
A ChAdOx1 nCoV-19 (Vaccine)

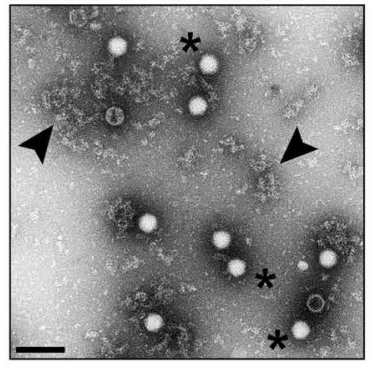

Supernatant

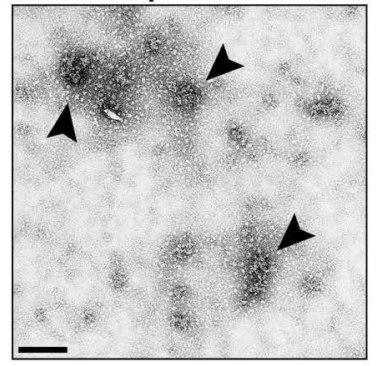

B

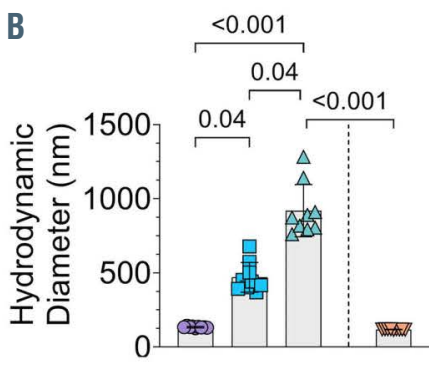

C
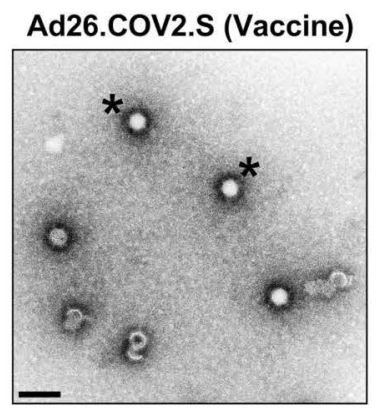

D
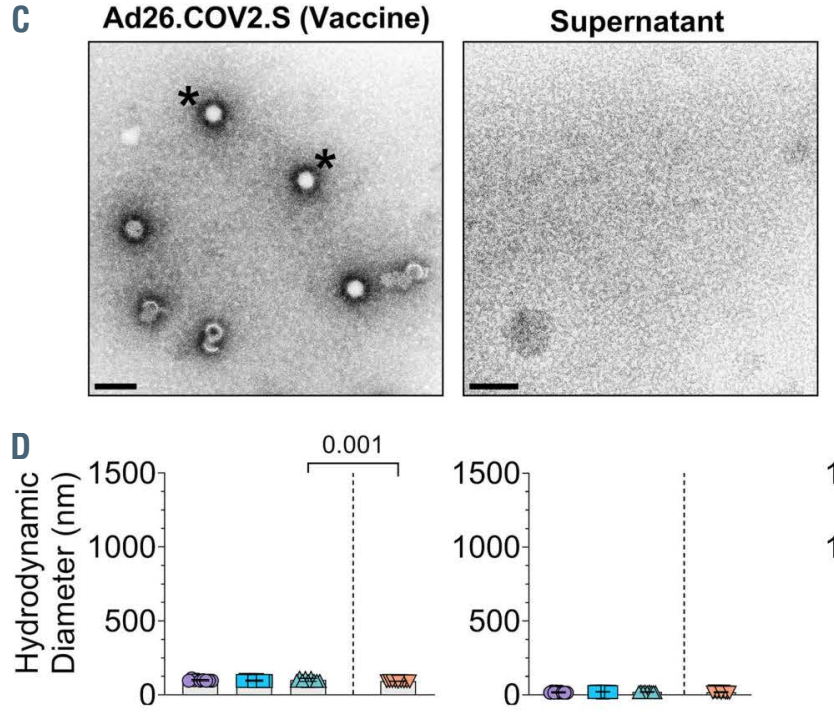
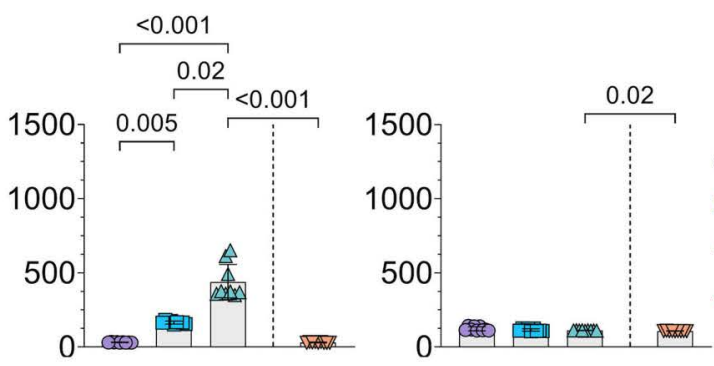

- Control

$\square+$ PF4 $(10 \mu \mathrm{g} / \mathrm{mL})$

$\triangle+$ PF4 $(50 \mu \mathrm{g} / \mathrm{mL})$

$\nabla+$ PF4 $(50 \mu \mathrm{g} / \mathrm{mL})$

$\nabla+\mathrm{UFH}(10 \mathrm{IU} / \mathrm{mL})$
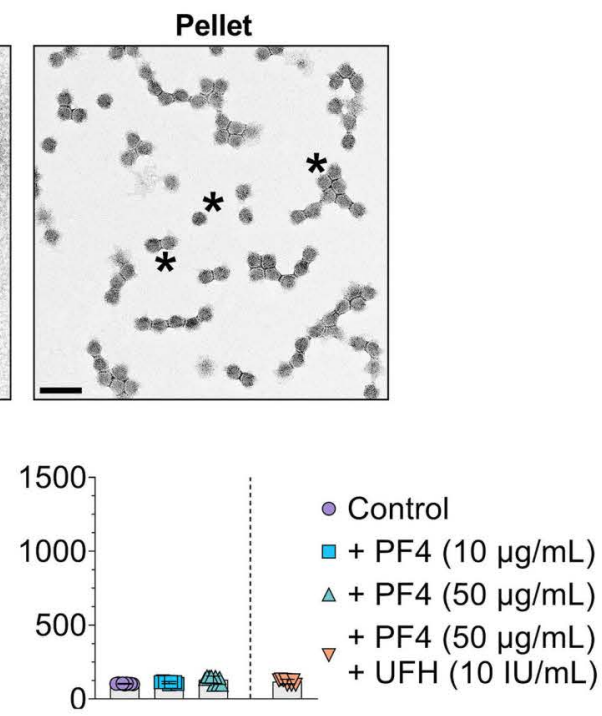

E ChAdOx1 nCoV-19 Ad26.COV2.S

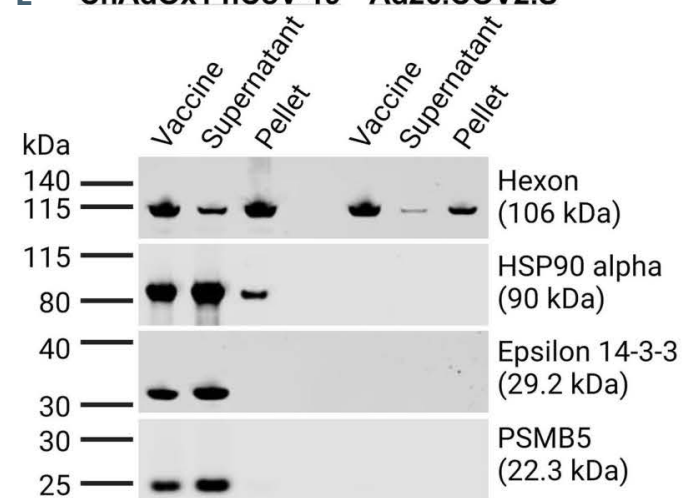

Figure 5. Analysis of vaccine components prepared by ultracentrifugation. (A, C) Representative transmission electron micrographs of (A) ChAdOx1 nCoV-19 and (C) Ad26.COV2.S vaccine and their respective supernatants and pellets obtained after ultracentrifugation. Asterisks and arrows indicate virions and electron dense amorphous vaccine components, respectively. Scale bar represents $200 \mathrm{~nm}$. (B, D) Changes in the hydrodynamic diameter (in nm) of (B) ChAdOx1 nCoV-19 and (D) Ad26.COV2.S vaccine and their respective supernatants and pellets obtained after ultracentrifugation of vaccines before and after addition of $10 \mu \mathrm{g} / \mathrm{mL}$ or $50 \mu \mathrm{g} / \mathrm{mL}$ platelet factor 4 (PF4) assessed by dynamic light scattering. Dissociation of complexes between PF4 and the vaccine component was achieved by the addition of unfractionated heparin (UFH; $10 \mathrm{IU} / \mathrm{mL}$ ) (E) Western blot analysis of vaccine and pellet or supernatant fraction is shown. Primary antibodies for viral vector (antihexon antibody) or human protein contaminants (anti-HSP90 antibody, anti-Epsilon 14-3-3 antibody, anti-PSMB5 antibody) were used. Statistical analysis was performed by one-way analysis of variance on ranks/Kruskal-Wallis followed by correction for multiple comparisons by two-stage Benjamini, Krieger, \& Yekutieli controlling the false discovery rate procedure $(n=9)$ and $\alpha<0.05$ was considered significant. 

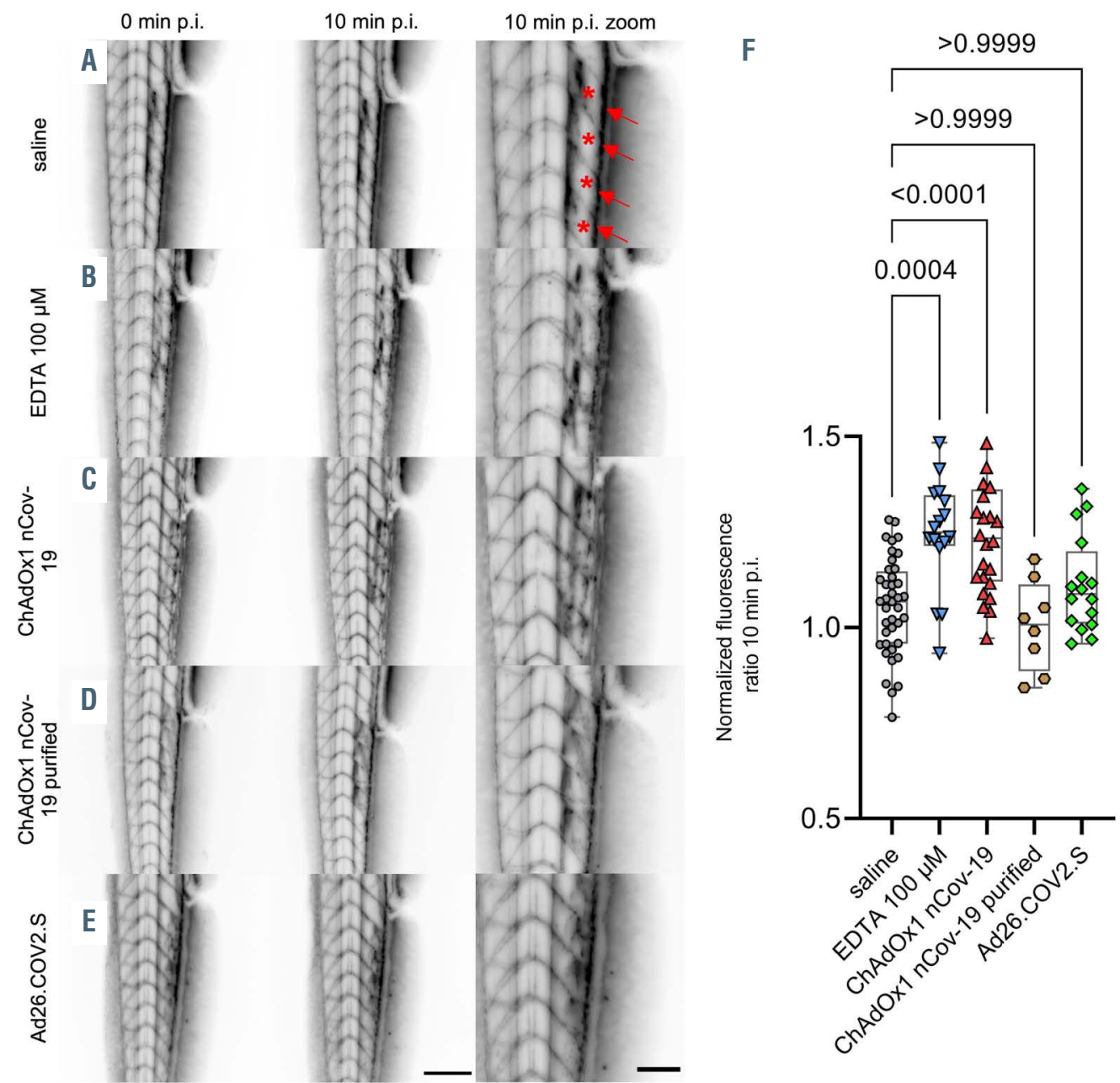

Figure 6. Vascular hyperpermeability assay. Five days post-fertilization Tg(fabp10a:gc-eGFP) zebrafish larvae were microinjected with either physiological saline, 100 $\mu$ M EDTA, ChAdOx1 nCoV-19, purified ChAdOx1 nCoV-19 or Ad26.COV2.S in four adjacent myotomes. Local fluorescence intensities of the myotomes were measured at $0 \mathrm{~min}$ and $10 \mathrm{~min}$ post-injection (p.i.) (red asterisks) and normalized to the respective intravascular fluorescence (red arrows). Injection of $100 \mu \mathrm{M}$ EDTA, as well as ChAdOx1 nCoV-19, resulted in a significantly elevated extravascular leakage of the $78 \mathrm{kDa}$ gc-eGFP compared to the saline control (A, B, C, F). However, injection of Ad26.COV2.S, purified ChAdOx1 nCoV-19 or physiological saline did not cause an increase of local vascular permeability (A, D, E, F). The scale bar in (E) represents $200 \mu \mathrm{m}$ without zoom and $100 \mu \mathrm{m}$ with zoom.

cofactor is needed. This cofactor is present in the supernatant of the ChAdOx1 nCoV-19 vaccine. However, this does not exclude complex formation of PF4 and Ad26.COV2.S in vivo. Several studies have shown the interaction of different adenoviruses with platelets and on the platelet surface they may also interact with PF4. In addition, hexon proteins or the virions may form complexes with PF4 when they come into contact with additional lymphatic or plasma proteins.

Beyond VITT/TTS, the potential for alloantibody formation by protein contaminants in vaccines might be a matter of concern since the PregSure ${ }^{\circledR}$ vaccine, used in cattle, induced alloantibody-driven bovine neonatal pancytopenia, a vaccine-induced alloimmune disease that was observed in young calves of PregSure ${ }^{\circledR}$-vaccinated cows and is characterized by hemorrhage, pancytopenia, and severe destruction of hematopoietic tissue. The plasma of cows that gave birth to affected calves contained alloantibodies, which were likely induced by alloantigenexpressing protein contaminants of the vaccine from the bovine kidney cell line used for vaccine production. ${ }^{29}$ Alloantibodies will only cause clinical effects in the case of pregnancy or organ transplantation. Systematic screening of vaccinated individuals excluding or confirming such alloantibodies should be performed to clarify whether this theoretical concern requires further measures.

Limitations of our study include its in vitro design. Furthermore, our findings do not exclude the contribution of certain cofactors (e.g., within the interstitial fluid, lymphatic system, plasma, or cell surfaces) to the induction of the anti-PF4 immune response. We also did not investigate involvement of $\mathrm{B}$-cell and $\mathrm{T}$-cell populations. Moreover, since both adenoviral vector-based vaccines have been discontinued in Germany, we cannot compare the immune responses among vaccinated individuals. Finally, the lack of a suitable in vivo model for induction of VITT/TTS limits the ability to reach definite conclusions regarding in vivo mechanisms.

In summary, we show that process-related impurities in the form of host-cell derived proteins, active proteases and unassembled hexon proteins differ in quality and quantity between ChAdOx1 nCoV-19 and Ad26.COV2.S SARS-CoV-2 vaccines. EDTA-induced capillary leakage 
and host-cell protein impurities might further facilitate induction of an anti-PF4 immune response by intravascular translocation of vaccine constituents and induction of an early inflammatory response after vaccination. These factors might explain the higher incidence rate of VITT/TTS for ChAdOx1 nCoV-19 compared to Ad26.COV2.S vaccines. However, the authors would like to point out again that only comprehensive vaccination of the human population can effectively contain the SARSCoV-2 pandemic.

\section{Disclosures}

$A G$ reports personal fees and non-financial support from Aspen, Boehringer Ingelheim, Instrumentation Laboratory, and Roche; grants from Ergomed, Rovi, Sagent, Portola, Fa. Blau Farmaceutics, Prosensa/Biomarin, DRK-BSD BadenWürtemberg/Hessen, and Biokit; personal fees from Bayer Vital, Chromatec, Sanofi-Aventis, and GTH e.V; grants and personal fees from Macopharma; as well as grants and other from DRK$B S D N S T O B$, In addition, AG reports having a patent, application n. 2021032220550000DE, pending.

\section{Contibutions}

$S M, F S, R P, K F, A R, U S, C C, J W, L S, C H, M G-S, M B$, $N E, A G$, and $U V$ conceived the research. $S M, F S, R P, K F, A R$, $U S, C C, J W, L S, C H, M G-S, M B, N E, A G$, and $U V$ defined the methodology. SM, FS, RP, KF, AR, MS, US, CC, JW, LS, $C H$, and $M G-S$. conducted the analysis. $S M, F S, R P, K F, A R$, US, CC., JW, LS, CH, MG-S, MB, NE, AG, and U.V. interpreted the data. $S M, F S, R P, K F, A R$, and $M S$ generated ways to visualize the data. $S M, F S, R P, K F, M B, N E, A G$, and $U V$ wrote the first draft of the manuscript. All authors contributed to the interpretation of results and manuscript editing. All authors approved the final version of the manuscript.

\section{Acknowledgments}

We thank Katrin Schoknecht for support in the proteomics analyses and Mandy Jörn for graphical design of electron microscopy micrographs.

\section{Funding}

This study was funded by Deutsche Forschungsgemeinschaft (DFG, German Research Foundation) grants: 374031971 - A06 and A11-TRR240, 398967434 - SFB/TR261, A11 - SFB877, P6 - KFO306, B8 - SFB841, and INST 2026/13-1 FUGG, the Ministerium für Wirtschaft, Arbeit und Gesundheit MecklenburgVorpommern (project COVIDPROTECT), "Structure and Function of the Proteasome System in Platelets" GR2232/8_1 and SE 885/2-1 (DFG), Leibniz WissenschaftsCampus ComBioCat - W10/2018, by the Federal Ministry of Education and Research (BMBF, grant 01GM1518B, STOP- FSGS), the Südmeyer fund for kidney and vascular research ("SïdmeyerStiftung für Nieren- und Gefäßforschung"), the Dr. Gerhard Büchtemann fund, Hamburg, Germany and the PeNe_C19 study by the Ministerium für Wirtschaft, Arbeit und Gesundheit Mecklenburg-Vorpommern.

\section{Data-sharing statement}

The mass spectrometry proteomics data have been deposited to ProteomeXchange (dataset identifier PXD027344).

\section{References}

1. Sadoff J, Gray G, Vandebosch A, et al. Safety and efficacy of single-dose Ad26.COV2.S vaccine against Covid-19. N Engl J Med. 2021;384(23):2187-2201.

2. Voysey M, Clemens SAC, Madhi SA, et al. Safety and efficacy of the ChAdOx1 nCoV19 vaccine (AZD1222) against SARS-CoV-2: an interim analysis of four randomised controlled trials in Brazil, South Africa, and the UK. Lancet. 2021;397(10269):99-111.

3. See I, Lale A, Marquez P, et al. Case series of thrombosis with thrombocytopenia syndrome following COVID-19 vaccinationUnited States, December 2020-August 2021. Available at: https://www.medrxiv.org/content/10.1101/ 2021.11.10.21266063v1.

4. Chevassut T, Hunt BJ, Pavord S. VITT, COVID-19 and the Expert Haematology Panel: the story of how the UK responded to emerging cases of vaccine-induced immune thrombocytopenia and thrombosis during the vaccination programme. Clin Med (Lond). 2021;21(6):e600-e602.

5. Paul-Ehrlich-Institut, Germany. Sicherheitsbericht: Verdachtsfälle von Nebenwirkungen und Impfkomplikationen nach Impfung zum Schutz vor COVID-19 seit Beginn der Impfkampagne am 27.12.2020 bis zum 30.09.2021 [accessed Dec 6, 2021]. Available at: https://www.pei.de/SharedDocs/Download s/DE/newsroom/dossiers/sicherheitsberichte/sicherheitsbericht-27-12-20-bis-3009-21.pdf? _blob=publicationFile \& $v=9$

6. Greinacher A, Thiele T, Warkentin TE,
Weisser K, Kyrle PA, Eichinger S. Thrombotic thrombocytopenia after ChAdOx1 nCov-19 vaccination. N Engl J Med. 2021;384(22):2092-2101.

7. Scully M, Singh D, Lown R, et al. Pathologic antibodies to platelet factor 4 after ChAdOx1 nCoV-19 vaccination. N Engl J Med. 2021;384(23):2202-2211.

8. Huynh A, Kelton JG, Arnold DM, Daka M, Nazy I. Antibody epitopes in vaccineinduced immune thrombotic thrombocytopaenia. Nature. 2021:596(7873):565-569.

9. Greinacher A, Selleng K, Palankar R, et al. Insights in ChAdOx1 nCoV-19 vaccineinduced immune thrombotic thrombocytopenia. Blood. 2021;138(22):2256-2268.

10. Krutzke L, Roesler R, Wiese S, Kochanek S. Process-related impurities in the ChAdOx1 nCov-19 vaccine [accessed May 4, 2021] Available https://www.researchsquare.com/article/rs477964/v1); DOI: 10.21203/rs.3.rs477964/v1.

11. Nickerson JL, Doucette AA. Rapid and quantitative protein precipitation for proteome analysis by mass spectrometry. J Proteome Res. 2020;19(5):2035-2042.

12. Blankenburg S, Hentschker C, Nagel A, et al. Improving proteome coverage for small sample amounts: an advanced method for proteomics approaches with low bacterial cell numbers. Proteomics. 2019;19(23):e1900192.

13. Perez-Riverol Y, Csordas A, Bai J, et al. The PRIDE database and related tools and resources in 2019: improving support for quantification data. Nucleic Acids Res. 2019;47(D1):D442-D450.

14. Berkers CR, Verdoes M, Lichtman E, et al.
Activity probe for in vivo profiling of the specificity of proteasome inhibitor bortezomib. Nat Methods. 2005;2(5):357-362.

15. Arastu-Kapur S, Anderl JL, Kraus M, et al. Nonproteasomal targets of the proteasome inhibitors bortezomib and carfilzomib: a link to clinical adverse events. Clin Cancer Res. 2011;17(9):2734-2743.

16. Provost A, Rousset C, Bourdon L, et al. Innovative particle standards and long-lived imaging for 2D and 3D dSTORM. Sci Rep. 2019;9(1):17967.

17. Laine RF, Tosheva KL, Gustafsson N, et al. NanoJ: a high-performance open-source super-resolution microscopy toolbox. J Phys D Appl Phys. 2019;52(16):163001

18. Hoboth P, Sebesta O, Sztacho M, Castano E, Hozák P. Dual-color dSTORM imaging and ThunderSTORM image reconstruction and analysis to study the spatial organization of the nuclear phosphatidylinositol phosphates. MethodsX. 2021;8:101372.

19. Schindelin J, Arganda-Carreras I, Frise E, et al. Fiji: an open-source platform for biologicalimage analysis. Nat Methods. 2012;9(7):676682.

20. Xie J, Farage E, Sugimoto M, Anand-Apte B. A novel transgenic zebrafish model for blood-brain and blood-retinal barrier development. BMC Dev Biol. 2010;10:76.

21. Siegerist F, Zhou W, Endlich K, Endlich N. $4 \mathrm{D}$ in vivo imaging of glomerular barrier function in a zebrafish podocyte injury model. Acta Physiol (Oxf). 2017;220(1):167173.

22. Baker AT, Boyd RJ, Sarkar D, et al. ChAdOx1 interacts with CAR and PF4 with implications for thrombosis with thrombocytopenia syndrome. Sci Adv. 2021;7(49):eabl8213. 
23. Eurpean Medicines Agency (EMA). Assessment report: COVID-19 Vaccine AstraZeneca [accessed Dec 6, 2021]. Available

at: https://www.ema.europa.eu/en/documents/assessment-report/vaxzevria-previously-covid-19-vaccine-astrazeneca-eparpublic-assessment-report_en.pdf.

24. Nicolai L, Leunig A, Pekayvaz K, et al. Thrombocytopenia and splenic platelet directed immune responses after intravenous ChAdOx1 nCov-19 administration [accessed July 29, 2021]. Available at: https://www.biorxiv.org/content/10.1101/2 021.06.29.450356v1.

25. Pfueller SL, Logan D, Tran TT, Bilston RA. Naturally occurring human IgG antibodies to intracellular and cytoskeletal components of human platelets. Clin Exp Immunol. 1990;79(3):367-373

26. Hauler F, Mallery DL, McEwan WA, Bidgood SR, James LC. AAA ATPase p97/VCP is essential for TRIM21-mediated virus neutralization. Proc Natl Acad Sci U S A. 2012;109(48):19733-19738.

27. Greinacher A, Selleng K, Mayerle J, et al.
Anti-platelet factor 4 antibodies causing VITT do not cross-react with SARS-CoV-2 spike protein. Blood. 2021;138(14):1269-1277.

28. Uzun G, Althaus K, Bakchoul T. No correlation between anti-PF4 and anti-SARS-CoV-2 antibodies after ChAdOx1 nCoV-19 vaccination. N Engl J Med. 2021;385(14):13341336.

29. Bastian M, Holsteg M, Hanke-Robinson $H$ Duchow K, Cussler K. Bovine neonatal pancytopenia: is this alloimmune syndrome caused by vaccine-induced alloreactive antibodies? Vaccine. 2011;29(32):5267-5275 